\title{
Desenvolvimento embrionário em ratas tratadas com Hypericum perforatum durante o período de implantação
}

\author{
F. Nepomuceno ${ }^{1}$, L. Las Casas ${ }^{2}$, V.M. Peters ${ }^{1}$, M.O. Guerra ${ }^{1 *}$ \\ ${ }^{1}$ Centro de Biologia da Reprodução, Universidade Federal de Juiz de Fora, Campus da UFJF, Cx. Postal 328, \\ 36001-970, Juiz de Fora, MG, Brasil, \\ ${ }^{2}$ Farmácia de Manipulação Las Casas, Gal. Pio X, Lj 174, 36010-001, Juiz de Fora, MG, Brasil
}

\begin{abstract}
RESUMO: O hipérico (Hypericum perforatum) é utilizado no tratamento alternativo da depressão, enfermidade que tem atingido mulheres, tanto no pós-parto quanto durante a gestação. Existem poucas informações sobre estudos experimentais quanto à toxicidade reprodutiva do hipérico. O presente trabalho tem por objetivo avaliar seu potencial embriotóxico. Ratas Wistar prenhes foram tratadas com 36 ou $360 \mathrm{mg} / \mathrm{kg}$ de extrato seco de Jarsin, por gavagem nos dias 5 e 7 pósinseminação. Animais do grupo controle receberam $0,5 \mathrm{~mL}$ de água destilada pela mesma via e dias. Após eutanásia no 150 dia, as seguintes variáveis foram analisadas: peso corporal materno e fetal, consumo de ração, sinais clínicos de toxicidade, peso de ovários e placentas, número de corpos lúteos, de reabsorções, de fetos vivos e de fetos mortos e proporção de implantação e de reabsorção. Não foram observadas diferenças significativas em nenhuma dessas variáveis, levando a concluir que no modelo experimental utilizado, o Hypericum perforatum não parece apresentar toxicidade para a mãe, não interfere na implantação do blastocisto e nem parece ser tóxico para o embrião.
\end{abstract}

Unitermos: Hypericum perforatum, implantação, desenvolvimento embrionário, extrato de jarsin, ratas, toxicidade.

\begin{abstract}
Embryo development in rats treated with Hypericum perforatum during the implantation period". Hypericum perforatum is used as an alternative treatment of depression, which is a disease that has been affecting women during post-partum or gestation. There is little information in experimental studies regarding the reproductive toxicity of hiperic. The present paper aims at assessing Hypericum perforatum's embryotoxic potential. Pregnant Wistar rats were treated with 36 or $360 \mathrm{mg} / \mathrm{kg}$ body weight of Jarsin dried extract, via oral gavage on days 5 and 7 post-insemination. Animals from the control group received $0.5 \mathrm{~mL}$ of distilled water through the same via and days. The animals were killed on the $15^{\text {th }}$ day and the following variables were analyzed: maternal and fetal body weight, food intake, clinical signs of toxicity, weight of ovaries and placenta, number of corpora lutea, resorptions, live and dead fetuses, and the proportion of implantation and resorption. No significant differences were observed in any of these variables, leading to the conclusion that in the experimental model used, Hypericum perforatum does not seem to be toxic to the mother, does not interfere with blastocyst implantation nor does it seem to be toxic to the embryo.
\end{abstract}

Keywords: Hypericum perforatum, implantation, embryo development, Jarsin extract, rats, toxicity.

\section{INTRODUÇÃO}

Além da depressão pós-parto, a depressão em mulheres gestantes é um fato conhecido e tem aumentado progressivamente nos últimos anos. Há relatos de que cerca de $70 \%$ de mulheres gestantes apresentam sintomas de depressão (Schmidt et al., 1997). Ela gera alteração no organismo materno, tanto por sua patologia própria quanto pelos fármacos usados para tratá-la (Newport et al., 2001).

Todos os fármacos conhecidos para tratamento da depressão cruzam a placenta, são observados no líquido amniótico, são excretados no leite (Newport et al., 2001; Hostetter et al., 2000) e não se sabe se ou como agem no organismo fetal.
O Hypericum perforatum L. (Gutiiferae), popularmente conhecido como hipérico, jarsin ou Erva-de-São-João, é utilizado há séculos em vários estados mórbidos mas, particularmente no tratamento de depressão leve a moderadamente grave (Simões, 2001; Linde et al., 2005). A vantagem do hipérico, quando comparado a outros antidepressivos sintéticos, é a baixa incidência de efeitos indesejáveis (Linde et al., 1996)

$\mathrm{O} H$. perforatum tem como princípio ativo a hipericina que, no extrato de Jarsin, encontra-se na concentração de 0,4\% (Harrer; Schulz, 1994; Wagner; Bladt, 1994). Estudos relacionados à toxicologia reprodutiva mostraram que a hipericina causou efeitos teratogênicos em embriões cultivados in vitro (Chan et al., 2001). Sua administração antes e durante a gestação 
de fêmeas de camundongos não afetou o desenvolvimento e nem a maturação física da prole (Rayburn et al., 2001). Entretanto, Gregoretti et al. (2004) relatam lesão hepática e renal em fetos de ratos, cujas mães foram expostas a hipericina durante a gestação e lactação. Quando a exposição ocorreu exclusivamente no período de organogênese, não causou malformações nem interferiu com o desenvolvimento fetal em ratos (Borges et al., 2005).

Não foram encontradas referências sobre estudos realizados no período de implantação do blastocisto. Nesta fase da gestação, a exposição da gestante a xenobióticos pode acarretar a morte do concepto sem que a mulher o perceba. A perda embrionária, não detectada nessa fase, pode induzir ao diagnóstico de infertilidade (Scially, 1992).

Considerando o risco de mulheres deprimidas fazerem uso inadvertido do hipérico em um período precoce de gestação, o presente trabalho teve por objetivo avaliar o potencial embriotóxico do extrato de Jarsin, administrado a ratas.

\section{MATERIAL E MÉTODOS}

Foram utilizadas 50 ratas (Rattus norvegicus) da linhagem Wistar, nuligestas, com cerca de quatro meses de idade, pesando aproximadamente 170 gramas, obtidas no biotério do Centro de Biologia da Reprodução da Universidade Federal de Juiz de Fora. Os animais foram mantidos em armários climatizados, com ração peletizada e água "ad libitum”.

As ratas foram acasaladas, ao final da tarde, com machos de fertilidade comprovada e a presença de espermatozóides no esfregaço vaginal, na manhã seguinte, foi indicativa do primeiro dia após inseminação (Keshri et al., 2003).

O Hypericum perforatum foi utilizado sob a forma de extrato seco de Jarsin (Lote SJ001108), fornecido pela Farmácia de Manipulação Las Casas (Juiz de Fora, MG, Brasil) que o adquiriu da empresa Galena Química Farmacêutica Ltda.

O trabalho foi realizado em duas etapas:

Experimento I: 20 ratas inseminadas foram distribuídas em dois grupos, controle e tratado, contendo 10 animais cada. Aos animais do grupo controle, foi administrado, via intragástrica, uma vez ao dia, às 9 horas, 0,5 mL de água destilada, durante os três dias que abrangem a implantação do blastocisto - 50 ao 7ํㅡ dias pós-inseminação - (Kanjanapothi et al., 1981; Souza et al., 1997). As ratas do grupo tratado receberam o extrato seco de Jarsin, contendo $36 \mathrm{mg} / \mathrm{kg}$ de peso corporal (equivalente à dose terapêutica humana - 1800mg/dia - Staffeldt, 1994) em 0,5 mL de água destilada, pela mesma via e durante os mesmos dias e horário que o grupo controle. O extrato foi administrado em uma dose/ dia de forma a evitar o manuseio excessivo do animal no início da prenhez, visto que tal manuseio, mesmo em procedimentos de rotina, causa aumento de temperatura, do cortisol e da prolactina circulantes (Balcombe, Barnard, Sandusky, 2004), fatores que podem interferir com o desenvolvimento embrionário.

Experimento II: 30 ratas inseminadas foram distribuídas em dois grupos, controle e tratado, contendo 15 animais cada. A administração foi realizada de forma idêntica ao experimento I para ambos os grupos, sendo que as ratas do grupo tratado receberam $360 \mathrm{mg} / \mathrm{kg}$ de peso corporal (dose 10 vezes superior à usada na terapêutica humana e semelhante à usada em ratos estressados por Trofimiuk et al., 2005) do extrato seco de Jarsin, em 0,5 $\mathrm{mL}$ de suspensão aquosa.

Para detectar a toxicidade materna obteve-se o peso corporal no primeiro dia pós-inseminação, no primeiro e último dia de tratamento ( $5^{-0}$ e $\left.7^{0}\right)$, no dia do sacrifício e após a remoção do sistema reprodutor. Foi feito o controle da estimativa de consumo diário de ração e foram observados ainda sinais clínicos indicativos de toxicidade, tais como piloereção, alteração no padrão de deambulação no interior da gaiola, ocorrência de diarréia e perdas sangüíneas vaginais (Christian, 2001).

Em ambos os experimentos, os animais foram sacrificados por exsangüinação total sob anestesia no 15ํdia pós-inseminação. Após laparotomia removeu-se todo o trato reprodutivo. Os ovários foram dissecados e pesados e seus corpos lúteos contados. Os cornos uterinos foram seccionados longitudinalmente e seu conteúdo examinado para contagem de fetos vivos (identificados pelo seu aspecto e batimentos cardíacos), reabsorções e fetos mortos. Os fetos e placentas foram removidos, limpos em papel de filtro umedecido e pesados. Cada feto foi observado sob microscópio estereoscópico para exame dos membros superiores e inferiores, fechamento de tubo neural e morfologia da face.

Foi calculada a proporção de implantação por grupo experimental: P.I. $=\left(\mathrm{n}^{\circ}\right.$ total de implantes $/ \mathrm{n}^{\circ}$ de corpos lúteos)x100 e a proporção de reabsorção: P.R. = (nº de reabsorções/ $n^{0}$ total de implantes)x100.

Os pesos corporais maternos nos dois grupos experimentais, os pesos corporais fetais e de placenta, assim como o consumo de ração, foram analisados através do teste de " $\mathrm{t}$ " de Student. Para comparar o peso corporal materno antes e depois do tratamento, utilizou-se o teste " $\mathrm{t}$ " de Student pareado. Os teste do Chi-quadrado ou Fisher exato foram usados para a análise das proporções de implantação e reabsorção. Nível de significância dos testes $\alpha=0,05$.

O protocolo experimental foi submetido e aprovado pelo Comitê de Ética na Experimentação Animal da Universidade Federal de Juiz de Fora (Protocolo $\mathrm{n}^{0}$ 38/2003).

\section{RESULTADOS}

Não foram detectadas piloereção, alterações na deambulação, diarréia ou perdas sangüíneas vaginais 
tanto nas ratas que receberam 36mg de extrato de Jarsin/ $\mathrm{kg}$ de peso corporal quanto nas que receberam $360 \mathrm{mg} / \mathrm{kg}$ de peso corporal.

A tabela 1 mostra o peso corporal materno, nos dias 1, 5, 7, 15 e após a remoção do aparelho reprodutor, nos experimentos I e II.

A comparação dos pesos corporais, ao longo do experimento, não mostrou diferença significativa entre os grupos controle e tratado. O peso corporal das ratas antes e depois do tratamento não apresentou diferença significativa $(\mathrm{p}>0,05)$.

O consumo de ração foi semelhante entre os grupos nos dois experimentos (resultados não apresentados).

A tabela 2 apresenta a proporção de implantação e a de reabsorção nos grupos controle e tratado de ambos experimentos.

As proporções de implantações e de reabsorções foram semelhantes entre os animais dos grupos controle e tratado $(\mathrm{p}>0,05)$.

A tabela 3 expressa o peso de ovários de ratas nos dois grupos experimentais. Não tendo ocorrido diferença significativa entre os grupos analisados ( $\mathrm{p}>0,05)$.

Não foram observadas alterações morfológicas externas fetais em nenhum dos grupos experimentais estudados.

Tabela 1. Peso corporal (g) de ratas controles e tratadas do experimento I e do experimento II.

\begin{tabular}{cccc}
\hline $\begin{array}{c}\text { Dias } \\
\text { Pós-inseminação }\end{array}$ & Grupo & Experimento I* & Experimento II** \\
& Controle & $(\mathrm{n}=10)$ & $169,50 \pm 10,29$ \\
\hline 1 & Tratado & $171,65 \pm 8,04$ & $167,10 \pm 10,35$ \\
& Controle & $167,70 \pm 8,57$ & $179,07 \pm 10,64$ \\
& Tratado & $180,25 \pm 8,93$ & $175,10 \pm 11,09$ \\
7 & Controle & $176,20 \pm 10,36$ & $182,57 \pm 11,70$ \\
& Tratado & $180,78 \pm 9,64$ & $177,27 \pm 11,29$ \\
15 & Controle & $180,20 \pm 10,89$ & $208,93 \pm 11,95$ \\
& Tratado & $203,50 \pm 11,32$ & $202,20 \pm 12,68$ \\
& Controle & $198,25 \pm 12,14$ & $195,86 \pm 12,16$ \\
& Tratado & $192,40 \pm 10,02$ & $190,00 \pm 12,16$ \\
\hline
\end{tabular}

Resultados expressos em média \pm desvio padrão (número de casos estudados).

*36 mg/kg e **360 mg/kg de peso corporal.***Peso corporal após remoção do trato reprodutor. p> 0,05.

Tabela 2. Proporção (\%) de implantação e de reabsorção nos grupos controle e tratado dos experimentos I e II.

\begin{tabular}{lcc}
\hline \multirow{2}{*}{ Experimentos } & \multicolumn{2}{c}{ Proporções (\%) } \\
\cline { 2 - 3 } & Implantação & Reabsorção \\
\hline Controle I & $85,5(100 / 117)$ & $7,0(7 / 100)$ \\
Tratado I* & $88,7(86 / 97)$ & $9,3(8 / 86)$ \\
Controle II & $82,4(145 / 176)$ & $3,4(5 / 145)$ \\
Tratado II** & $86,6(149 / 172)$ & $2,7(4 / 149)$ \\
\hline
\end{tabular}

*36 mg/kg e **360 mg/kg de peso corporal.

Tabela 3. Peso dos ovários de ratas controles e tratadas dos experimentos I e II.

\begin{tabular}{lcc}
\hline & Experimentos & Peso dos ovários (2) \\
\hline Controle I & $65,0 \pm 12,5(10)$ \\
Tratado I* & $59,7 \pm 11,2(9)^{* * *}$ \\
Controle II & $65,1 \pm 5,3(15)$ \\
Tratado II** & $63,7 \pm 9,1(15)$ \\
\hline
\end{tabular}

Resultados expressos em média \pm desvio padrão (número de casos estudados).

* $36 \mathrm{mg} / \mathrm{kg}$; **360 mg/kg de peso corporal; *** Um dado foi eliminado (“outlier”). 
Tabela 4. Peso médio (mg)/ninhada de fetos e placentas nos experimentos I e II.

\begin{tabular}{lcc}
\hline \multirow{2}{*}{ Experimento } & \multicolumn{2}{c}{ Peso } \\
\cline { 2 - 3 } & Fetos & Placentas \\
\hline Controle I & $159,8 \pm 9,9(10)$ & $149,7 \pm 12,8(10)$ \\
Tratado I* & $162,4 \pm 14,7(10)$ & $156,5 \pm 15,1(10)$ \\
Controle II & $162,5 \pm 14,2(15)$ & $154,2 \pm 20,6(15)$ \\
Tratado II** & $161,5 \pm 10,7(15)$ & $144,7 \pm 10,4(15)$ \\
\hline
\end{tabular}

Resultados expressos em média \pm desvio padrão (número de casos estudados).

* $36 \mathrm{mg} / \mathrm{kg}$ e **360 mg/kg de peso corporal.

Na tabela 4, podem ser verificados os pesos médios dos fetos e das placentas, por ninhada, nos experimentos I e II.

Não houve diferença significativa nos pesos de fetos e placentas entre os grupos $(p>0,05)$.

\section{DISCUSSÃO}

Alterações no organismo materno perturbam o desenvolvimento embrionário conforme já demonstrado por Khera que, em 1985, havia correlacionado malformações fetais que ocorrem com baixa freqüência (exencefalia, encefalocele, micro ou anoftalmia e outras) à redução do peso materno. Embora essa relação tenha sido contestada por Chahoud et al. (1999), não existem dúvidas de que a toxicidade materna pode influir no bom desenvolvimento do embrião e Scially (1992) sugere que não existe sentido analisar efeitos sobre o feto, quando há manifesta toxicidade materna.

Os indicativos de toxicidade materna habitualmente observados em ratas são: alterações no peso corporal, de consumo de alimento e água, alteração da deambulação na gaiola, piloereção, diarréia, mortalidade materna, perdas sangüíneas vaginais (Manson; Kang, 1994; Chahoud et al., 1999). Como não foram observadas alterações nos dados clínicos acima mencionados em nenhum dos grupos estudados, pode-se supor que a administração do extrato de Jarsin às mães não causou toxicidade, observável por critérios clínicos.

As condições hormonais maternas também são necessárias para o bom desenvolvimento embrionário, sendo amplamente conhecido que níveis sangüíneos de progesterona inadequados interferem com a viabilidade do embrião, por não permitirem que o endométrio esteja adequadamente preparado para a sustentação da gestação.

Através do peso de ovário e do número de corpos lúteos de gestação pode-se deduzir, indiretamente, as condições hormonais relativas a progesterona materna. O peso do ovário depende bastante do número e do volume dos corpos lúteos, visto serem eles as maiores estruturas no ovário (Waynforth, 1971). Os corpos lúteos são os principais responsáveis pela secreção de progesterona (Kato et al., 1979); eles aumentam de volume durante a gestação, tendo sido demonstrado que seu crescimento está intimamente correlacionado com o aumento de secreção de progesterona e 20-hidroxi-progesterona (Uchida et al., 1970), hormônios também indispensáveis à manutenção da prenhez na rata.

Nos dois grupos experimentais foram encontrados pesos de ovários e número de corpos lúteos semelhantes, o que permite supor que o ambiente hormonal materno não diferiu entre eles.

A proporção de implantação correlaciona o número de locais de implantação e o número de corpos lúteos (Kato et al., 1979); quanto maior a proporção, maior o número de ovulações que resultaram em oócitos fertilizados e blastocistos implantados. Como não se observaram diferenças significativas entre os grupos experimentais quanto à proporção de implantação dos blastocistos parece razoável supor que o extrato de Jarsin não alterou o processo de implantação.

A partir da implantação, o embrião pode continuar seu desenvolvimento normal, desenvolver-se anormalmente ou morrer. Reabsorção é o nome que se dá para a lise 'in situ' de um embrião ou feto (Kalter, 1980). A proporção de reabsorções correlaciona o número de reabsorções observadas e o número de implantes viáveis (os que contêm um feto vivo) - quanto maior a proporção de reabsorções maior, evidentemente, o número de fetos cujo desenvolvimento foi interrompido.

Não se observaram diferenças significativas quanto à proporção de reabsorções nos grupos experimentais estudados, o que leva à suposição de que a administração do extrato de Jarsin não interferiu com o progresso do desenvolvimento embrionário após a implantação. Tal suposição parece ser corroborada pelo peso corporal e de placenta, semelhantes nos dois grupos, visto que o crescimento normal dos fetos depende de uma integração complexa entre fatores genéticos, imunológicos, endocrinológicos, nutricionais, vasculares e de fatores ambientais. Alterações em quaisquer desses fatores interrompe o crescimento e o desenvolvimento normal do embrião/feto (Chahoud et al., 1999).

Tomados em conjunto, os dados acima mencionados parecem indicar ausência de efeitos tóxicos maternos e embriotóxicos do hipérico, administrado sob a forma de extrato de Jarsin, durante o período de implantação do blastocisto em ratas.

\section{CONCLUSÃO}


O Hypericum perforatum, administrado a ratas Wistar tanto na dose de $36 \mathrm{mg} / \mathrm{kg}$ quanto na de $360 \mathrm{mg} / \mathrm{kg}$ de peso corporal, não parece apresentar toxicidade para a mãe, não interfere na implantação do blastocisto e nem parece ter potencial tóxico para o embrião.

\section{AGRADECIMENTOS}

Ao acadêmico Eduardo Siqueira Fernandes e à mestranda Luciana Valente Borges pela paciente e cuidadosa formatação, revisão do texto e versão do resumo para a língua inglesa. À Profa. Dra. Rita de Cássia da Silveira e Sá pela correção do “abstract”.

\section{REFERÊNCIAS}

Balcombe JP, Barnard ND, Sandusky C 2004. Laboratory routines cause animal stress. Contemporary Topics 43: 42-51.

Borges LV, Peters VM, Las Casas L, Guerra MO 2005. Avaliação da toxicidade do Hypericum perforatum administrado a ratas prenhes. Rev Ass Méd Bras 25 (3) In press.

Chan LY, Chiu P, Lau T 2001. A study of hypericin-induced teratogenicity during organogenesis using a whole rat embryo culture model. Fertil Steril 76: 1073-1074.

Chahoud I, Ligensa A, Dietzel L, Faqi AS 1999. Correlation between maternal toxicity and embryo/fetal effects. Reprod Toxicol 13: 375-381.

Christian MS 2001. Test methods for assessing female reproductive and developmental toxicology. cap. 29. In: Hayes, W. Method of toxicology. Philadelphia: Taylor \& Francis, p.1301-1381.

Gleich J, Froberg H 1997. General teratological techniques. In: Neubert D, Merker HJ, Kwasigroch TE. Methods in prenatal toxicology. Massachusetts: PSG Publishing Company, p.94-102.

Gregoretti B, Stebel M, Candussio L, Crivellato E, Bartoli F, Decorti G 2004. Toxicity of Hypericum perfuratum (St. John`s wort) administered during pregnancy and lactation. Toxicol Appl Pharmacol 200: 201-205.

Guerra MO, Peters VM 1999. Como um mamífero se desenvolve desde a fertilização até a implantação do blastocisto. Boletim do Centro de Biologia da Reprodução UFJF 18: $15-32$.

Hafez ESE 1970. Reproduction and breeding techniques for laboratory animals. Philadelphia: Lea \& Febiger, p.375.

Harrer G, Schulz V 1994. Clinical investigation of the antidepressant effectiveness of Hypericum. J Geriatr Psych Neur 7 (suppl. 1): 6-8.

Hostetter A, Ritchie JC, Stowe ZN 2000. Amniotic fluid and umbilical cord blood concentrations of antidepressants in three women. Biol Psychiatry 48: 1032-1034.

Kalter H 1980. The relationship between congenital malformations and prenatal mortality in experimental animals. In: Potter I.(ed), Hook EB. Human embryonic and fetal death. New York: Academic Press, p.29-44.

Kanjanapothi D, Smitasiri Y, Panthong A, Taesotikul T, Rattanapanone V 1981. Post coital antifertility effects of Menta arvensis. Contraception 24: 559-568.

Kato H, Morishige WK, Rothchild I 1979. A quantitative relationship between the experimentally determined number of conceptuses and corpus luteum activity in pregnant rat. Endocrinology 105: 846-850.

Keshri G, Lakshmi V, Singh MM 2003. Pregnancy interceptive activity of Melia azedarach Linn. in adult female Sprague-Dawley rats. Contraception 68: 303-306.

Khera KS 1985. Maternal toxicity: a possible etiological factor in embryo-fetal deaths and fetal malformations of rodent-rabbit species. Teratology 31: 129-153.

Khera KS 1987. Maternal toxicity of drugs and metabolic disorders a possible etiologic factor in the intrauterine death and congenital malformation: a critique on human data. CRC Crit Rev Toxicol 17: 345-375.

Lemônica IP 1996. Embriofetotoxicidade. In: Oga S. Fundamentos de toxicologia. São Paulo: Atheneu, p.12-94.

Linde K, Ramirez G, Mulrow CD, Pauls A, Weidenhammer W, Melchart D 1996. St. John's wort for depression - an overview and meta-analysis of randomized clinical trials. Brit Med J 313: 253-258.

Linde K, Berner M, Egger M, Mulrow C 2005. St. John' wort for depression - an overview and meta-analysis of randomized controlled trials. Brit J Psychiat 186: 99107.

Manson JM, Kang YJ. Test methods for assessing female reproductive and developmental toxicology. In: Hayes AW 1994. Principles and methods of toxicology. 3.ed. New York: Raven Press. Cap.28, p. 989-1037.

Moore KL, Persaud TVN 2000. Embriologia clínica. 6 ed. Rio de Janeiro: Guanabara Koogan.

Newport DJ, Wilcox MM, Stowe ZN 2001. Antidepressants during pregnancy and lactation: defining exposure and treatment issues. Seminars in Perinatology 25: 177-190.

Rayburn WF, Gonzalez CL, Christensen HD, Stewart JD 2001. Effect of prenatally administered Hypericum (St. John`s wort) on growth and physical maturation of mouse offspring. Am J Obst Gynecol 184: 191-195.

Simões CMO et al. 2001. Farmacognosia: da planta ao medicamento. 3.ed. Porto Alegre: Ed. da Universidade/ UFRGS e Ed. da UFSC.

Souza ER, Guerra MO, Peters VM 1997. Desenvolvimento de pré-embriões de ratas Wistar da colônia do Biotério do Centro de Biologia da Reprodução. Boletim do Centro de Biologia da Reprodução UFJF 16: 63-70.

Sttafeldt B 1994. Pharmacokinetics of hypericin and pseudohypericin after oral intake of the Hypericum perforatum extract LI 160 in healthy volunteers. $J$ Geriatr Psychiatry Neurol 7: S47-53.

Trofimiuk E, Walesiuk A, Braszko JJ 2005. St. John's wort (Hypericum perfuratum) diminished cognitive impairment caused by restrictive stress in rats. Pharmacol Res 51: 239-246.

Wagner H, Bladt S 1994. Pharmaceutical quality of Hypericum extracts. J Geriatr Psych Neur 7 (suppl.1): 65-68.

Waynforth HB 1971. Changes in the volume of rats corpus luteum during pregnancy and after surgical interference with the uterus and placenta. Acta Endocrinol 66: 296302.

Uchida K, Kadowaki M, Nomura Y, Myata K, Miyake T, 1970. Relationship between ovarian progestin secretion and corpora lutea function in pregnant rat. Endocri Jpn 17: 499-507. 